\title{
ESTADO ATUAL DO DIAGNÓSTICO E TRATAMENTO DA APENDICITE AGUDA NA CRIANÇA: AVALIAÇÃO DE 300 CASOS
}

\author{
PRESENT STATUS FOR DIAGNOSIS AND TREATMENT OF ACUTE APPENDICITIS IN \\ CHILDREN: EVALUATION OF 300 CASES
}

\author{
Pedro Félix Vital Jr¹; José Luiz Martins, TCBC-SP²
}

\begin{abstract}
RESUMO: Objetivos: Avaliar o estado atual do diagnóstico e tratamento da apendicite aguda em crianças operadas em dois grandes hospitais quaternários da cidade de São Paulo, no período de 30 meses. Método: Nossa casuística constou de 300 crianças operadas por apendicite aguda no período de 1998 a 2000 (65\% do sexo masculino e $35 \%$ feminino). Foram analisadas as variáveis idade, sexo, manifestações clínicas, tempo gasto para o diagnóstico, achados de exame físico, laboratoriais e cirúrgicos, antimicrobianos administrados, complicações pós-operatórias e tempo de internação. Utilizou-se o teste t de Student para avaliar duas variantes e Análise de Variâncias quando mais de duas. Resultados: Diagnosticou-se inicialmente apendicite aguda em apenas $63 \%$ dos casos, tendo os $35 \%$ restantes, diagnóstico de abdome agudo cirúrgico. O tempo decorrido na realização do diagnóstico foi superior a 24 horas em $57,4 \%$ dos casos, denotando retardo importante na sua elaboração. Dor abdominal $(85,3 \%)$ e irritação peritoneal ( $82 \%)$ em fossa ilíaca direita foram os sinais e sintomas mais freqüentes. Identificou-se leucocitose em $83 \%$ dos pacientes e leucocitúria em $39,7 \%$. Em 92,4\% das radiografias simples de abdome encontramos imagens sugestivas de apendicite aguda. A ultra-sonografia abdominal foi diagnóstica em $80,1 \%$ dos casos. Utilizaram-se esquemas antimicrobianos especialmente para agentes gram-negativos e anaeróbicos. A principal complicação foi infecção da ferida cirúrgica, não tendo sido observada mortalidade no grupo. A média de internação foi de 5.2 e 6,0 dias para meninos e meninas respectivamente. Conclusão: Mesmo com melhor conhecimento sobre apendicite aguda, refinamento técnico, laboratorial, radiológico e uso de antibioticoterapia adequada, o tempo de para diagnóstico e a morbidade ainda se mantém alta na idade pediátrica (Rev. Col. Bras. Cir. 2005; 32(6): 310-315).
\end{abstract}

Descritores: Apendicite; Doença aguda; Apendicite/diagnóstico; Abdome agudo. Técnica de diagnóstico do sistema digestório; Complicações; Criança.

\section{INTRODUÇÃO}

Apendicite aguda é a doença que mais comumente requer cirurgia abdominal de emergência na criança, constituindo a principal causa de abdômen agudo cirúrgico na criança maior de dois anos de idade, e responsável por cerca de $10 \%$ de todas as admissões em salas de emergências pediátricas ${ }^{1,2}$. Em nossa prática clínica diária temos observado que, apesar dos grandes avanços tecnológicos nos métodos auxiliares ao diagnóstico, não houve redução significante da morbidade do paciente com apendicite aguda na idade pediátrica.

Deste modo, resolvemos avaliar o comportamento clínico da apendicite aguda na criança, o estado atual do seu diagnóstico, eficácia do tratamento e morbi-mortalidade em nosso meio.

\section{MÉTODO}

Estudamos prospectivamente 300 pacientes operados de apendicite aguda em dois grandes Hospitais de referência quaternária da cidade de São Paulo, Hospital São Paulo e Hospital Santa Marcelina, num período de 30 meses, entre 1998 a 2000.

Os pacientes tinham faixas etárias entre um e 13 anos, eram atendidos inicialmente por pediatras, e posteriormente solicitadas avaliações da cirurgia pediátrica, quando eram incluídos em protocolo onde se analisava a idade, sexo, manifestações clínicas, achados de exame físico, exames laboratoriais (hemograma e análise de urina), método de diagnóstico por imagem (raio x simples de abdome ortostático e em decúbito dorsal e ultra-sonografia abdominal), tempo gasto entre o início do quadro clínico e o diagnóstico, estado evolutivo da doença, classificação macroscópica do apêndice cecal ( edematosa, flegmonosa e gangrenosa), tempo de permanência em íleo paralítico, local de exteriorização dos drenos ( contra-abertura ou pela ferida cirúrgica), tempo de manutenção de drenos, persistência do quadro febril, antimicrobianos administrados, complicações pós-operatórias e tempo de permanência em internação hospitalar.

Quando as médias das variáveis eram comparadas entre si utilizou-se o teste estatístico t de Student. No entanto, quando mais de duas variáveis eram comparadas, a técnica de Análise de Variância foi aplicada. Fixou-se como nível

1. Mestre em Cirurgia Pediátrica pela Universidade Federal de São Paulo - Escola Paulista de Medicina; Cirurgião Pediátrico da Disciplina de Cirurgia Pediátrica da Universidade Federal de São Paulo- Escola Paulista de Medicina e do Hospital Santa Marcelina.

2. Professor Adjunto - Livre-Docente da Disciplina de Cirurgia Pediátrica da Universidade Federal de São Paulo - Escola Paulista de Medicina e Chefe do Serviço de Cirurgia Pediátrica do Hospital Santa Marcelina.

Recebido em 21/07/2005

Aceito para publicação em 27/09/2005

Conflito de interesse: nenhum

Fonte de financiamento : nenhuma

Trabalho realizado nos Hospitais São Paulo e Santa Marcelina da Cidade de São Paulo. 
de rejeição de hipótese de nulidade o valor menor ou igual a $0,05 \%$.

\section{RESULTADOS} na Tabela 1

As características gerais da análise podem ser vistas

As manifestações clínicas mais encontradas foram a dor em fossa ilíaca direita $(83,5 \%)$, náuseas e vômitos $(80,3 \%)$, anorexia $(61,6 \%)$, dor generalizada $(34,7 \%)$ e parada na eliminação de gases e fezes $(28,3 \%)$. Dentre os sinais clínicos chamou atenção a descompressão brusca positiva (82\%), febre (68\%), desidratação (55\%), diferencial axilo/retal $(33,3 \%)$, descompressão brusca generalizada (18\%) e toque retal doloroso $(15 \%)$.

Ao relacionarmos os resultados dos exames laboratoriais observamos que o hemograma foi realizado em $97,3 \%$ dos casos, encontrando-se normal em $12 \%$ dos pacientes. Havia leucocitose em $83,9 \%$, leucopenia em $4,1 \%$ e desvio para esquerda em $77,2 \%$.

A análise de urina foi indicada em $85,7 \%$ das crianças, encontrando-se normal em 60,3\%. Leucocitúria foi observada em $39,7 \%$.

Os resultados relacionados aos exames de imagens estão indicados na Tabela 2.

Administrou-se um único tipo de antimicrobiano em $21 \%$ dos pacientes enquanto que mais de um em $79 \%$.Os mais utilizados foram: metronidazol $(81,7 \%)$, amicacina $(68,7 \%)$, gentamicina $(6,7 \%)$, cefalosporinas $(49 \%)$, Deste grupo a cefalotina, ceftriaxona e a cefalexina foram os mais freqüentemente indicados.

Os procedimentos cirúrgicos e achados operatórios são analisados na Tabela 3 .

Quanto à evolução pós-operatória verificamos que $71 \%$ dos doentes permaneceram em íleo adinâmico por mais de 13 horas. Cerca de $51,7 \%$ dos drenos de penrose foram mantidos por período de quatro a seis dias e o quadro de febre recorrente foi observado por até 48 horas em 68,2\% dos casos.

A frequiência das complicações pós-operatórias assim como a relação entre sexo, idade e tempo de hospitalização, presença de coleções intraperitoneais e evolução anátomopatológica do apêndice cecal são mostrados nas Tabelas 4 a 7 .

\section{DISCUSSÃO}

A apendicite aguda é uma das principais causas de hospitalização de crianças entre um e 14 anos, e embora exista atualmente um acentuado decréscimo da mortalidade relatada, associa-se a índice de morbidade que se mantém alto $^{3,4}$. O seu diagnóstico na idade pediátrica pode ser particularmente difícil, principalmente nos estágios mais iniciais da doença, pelas variações anatômicas em suas localizações, assim como nas menores faixas etárias ${ }^{5}$. A prática médica diária tem demonstrado que o diagnóstico dessa afecção na criança ainda tem sua maior base no quadro clínico e exame físico. Em nossa casuística, observamos que o tempo decorrido entre a admissão e o diagnóstico foi
Tabela 1 - Características gerais da casuística.

\begin{tabular}{lcc}
\hline Variáveis & $\mathbf{N}$ & $\%$ \\
\hline Sexo & & \\
$\quad$ Masculino & 195 & 65,0 \\
$\quad$ Feminino & 105 & 35,0 \\
Idade & & \\
$\quad$ Até 7 anos & 114 & 38,0 \\
$\quad 8$ a 10 anos 133 & 44,3 & \\
$\quad$ Mais de 10 anos & 53 & 17,7 \\
Tempo entre o início da doença & & \\
e o diagnóstico & & \\
$\quad$ Menor que 24 horas & 33 & 11 \\
24 a 48 horas & 119 & 39,7 \\
$\quad$ 48 a 72 horas & 103 & 34,4 \\
$\quad$ Mais de 72 horas & 45 & 15 \\
Diagnóstico inicial & & \\
$\quad$ Apendicite aguda & 187 & 62,3 \\
$\quad$ Abdome agudo inflamatório & 109 & 36,3 \\
$\quad$ Outros & 4 & 1,3 \\
\hline
\end{tabular}

Tabela 2 - Avaliação radiológica e ultra-sonográfica.

\begin{tabular}{lrl}
\hline Exame & $\mathbf{N}$ & $\%$ \\
\hline Radiografia simples de abdome & 287 & 95,7 \\
Íleo paralítico & 262 & $91,3^{* *}$ \\
Psoas não visualizado & 177 & $61,7 * *$ \\
Escoliose & 140 & $48,8^{* *}$ \\
Fecalito & 51 & 17,8 \\
Pneumoapendicograma & 2 & $0,7^{* *}$ \\
Normal & 10 & $3,3^{* *}$ \\
Ultra-sonografia de abdome & 156 & 52,0 \\
Positiva para apendicite & 125 & $80,1 *$ \\
Normal & 31 & $19,9 *$ \\
Radiografia simples de tórax & 43 & 14,3 \\
\hline
\end{tabular}

** percentual com base no № de radiografias de abdome realizadas. * percentual com base no № de ultra-sonografias de abdome realizadas.

superior a 49 horas em cerca de $49,4 \%$ dos casos, tendo sido feito o diagnóstico de apendicite aguda em apenas $62,3 \%$ dos pacientes. Chamou a nossa atenção o fato de que, apesar de toda a evolução atual no que diz respeito principalmente aos meios diagnósticos por imagem, ainda existem grandes dificuldades no diagnóstico precoce refletidos pelos inúmeros casos operados com coleções purulentas intra-abdominais $(50,3 \%)$, além dos pacientes que evoluíram com complicações pós-operatórias, 30,3\%, com um número elevado de reoperações (22\%).

Relacionamos os fatos acima mencionados à uma desatenção inicialmente domiciliar às queixas das crianças principalmente na idade pré-escolar, associado a dificuldades no reconhecimento de quadros clínicos menos característicos por parte de alguns profissionais e por fim ao difícil acesso à saúde pública. Essas dificuldades tornaram-se mais evidentes 
Tabela 3 - Procedimentos cirúrgicos e achados intra-operatórios.

\begin{tabular}{lrc}
\hline $\begin{array}{l}\text { Procedimentos cirúrgicos e achados } \\
\text { intra-operatórios }\end{array}$ & N & \% \\
\hline Tipos de incisões & & \\
$\quad$ Transversa & 295 & 98,3 \\
$\quad$ Mc Burney & 3 & 1,0 \\
$\quad$ Paramediana & 2 & 0,7 \\
Presença de pús na cavidade peritoneal & 151 & 50,3 \\
Fase evolutiva da doença & & \\
$\quad$ Edematosa & 25 & 8,3 \\
$\quad$ Flegmonosa & 212 & 70,7 \\
$\quad 63$ & 21,0 \\
Gangrenosa & 84 & 28 \\
Perfuração do apêndice cecal & 6 & 2,0 \\
Pesquisa de divertículo de Meckel & & \\
Tratamento do coto apendicular & 291 & 97 \\
$\quad$ Oschner & 6 & 2,0 \\
$\quad$ Parkerker & 147 & 49 \\
Drenagem da cavidade peritoneal & 114 & $77,6^{*}$ \\
$\quad$ Por contra-abertura & 33 & $22,5^{*}$ \\
$\quad$ Pela ferida operatória & 31 & 10,3 \\
Lavagem da cavidade peritoneal & 198 & 66 \\
Limpeza mecânica & & \\
\hline
\end{tabular}

* percentual com base no $n^{-}$de casos drenados.

em pacientes abaixo de três anos de idade, onde chamava a atenção à presença situações clínicas mais sistêmicas com graus variados de desidratação e toxemia associados à distensão abdominal, ocasionalmente com complicações respiratórias, e pré-adolescentes do sexo feminino, quando as manifestações anexiais foram aventadas.

Alguns autores acreditam que retardo no diagnóstico e indicação cirúrgica por período de até 12 horas, em pacientes com quadros infecciosos controlados não determinam piora no prognóstico desses doentes, podendo segundo eles, realizar o procedimento cirúrgicos em horários mais adequados ${ }^{6}$.

Quanto ao sexo, encontramos uma predominância do masculino (65\%), com média de idade de 8,04 entre os meninos e 8,05 entre as meninas. Várias publicações ${ }^{7-11}$ tem relacionado sexo e idade com o grau de gravidade da doença e erros diagnósticos.
Tabela 4 - Complicações pós-operatórias.

\begin{tabular}{lcc}
\hline Complicações & N & \% \\
\hline Total de complicações & 91 & 30,3 \\
Infecção de ferida cirúrgica & 45 & $49,5^{*}$ \\
Abscessos intracavitários & 9 & $9,9^{*}$ \\
Abdome agudo obstrutivo & 7 & $7,7^{*}$ \\
Broncopneumonias & 5 & $5,5^{*}$ \\
Outras & 5 & $5,5^{*}$ \\
Reoperações & 20 & 22 \\
\hline
\end{tabular}

* percentual com base nos 91 casos com complicações.

Dentre as manifestações clínicas as mais freqüentes foram a dor em fossa ilíaca direita, náuseas, vômitos e anorexia. Algumas vezes a característica da dor diferiu quanto ao sexo, sendo mais localizada no sexo masculino. Nas meninas na idade pré-menstrual houve maior dificuldade de caracterização do quadro doloroso abdominal.

Os sinais mais freqüentemente encontrados ao exame físico foram a irritação peritoneal em fossa ilíaca direita (82\%), febre (68\%) e desidratação (55\%). O toque retal foi útil em $15 \%$ dos pacientes, em função das dificuldades na sua realização inerentes a idade em questão, afastando em alguns casos a presença de abscessos de pequena bacia além de patologias anexais em meninas com idades maiores. Nos casos suspeitos, sempre que possível frente à situação clínica encontrada, foram realizados exames de imagens adequados, principalmente os estudos radiológicos simples e a ultrasonografia.

Um outro problema comum relacionado ao retardo no diagnóstico encontra-se na interpretação errônea de exames laboratoriais. Esse fato pode aumentar o número de casos que evoluem com perfurações dos apêndices cecais, formações de abscessos intra-peritoneais e aumento da morbi-mortalidade. Nenhum dado clínico, laboratorial ou de imagem confere $100 \%$ de acurácia ao diagnóstico sendo muito importante a elaboração cuidadosa da história clínica e exame físico dos pacientes ${ }^{12,13}$. Encontramos publicações relacionando achados clínicos de irritação peritoneal associada à leucocitose e temperatura igual ou superior a $38^{\circ}$ a maior probabilidade de a apendicite em $85 \%$ dos ca$\operatorname{sos}^{14}$.

Tabela 5 - Relação entre o sexo, idade e tempo de hospitalização.

\begin{tabular}{l|cc|c}
\hline Variável & \multicolumn{2}{|c|}{ Sexo } & Teste t de Student (p) \\
\hline & $\begin{array}{c}\text { Masculino } \\
(\mathrm{n}=195)\end{array}$ & $\begin{array}{c}\text { Feminino } \\
(\mathrm{n}=105)\end{array}$ \\
& $\begin{array}{c}\text { média } \\
(\mathrm{dp})\end{array}$ & $\begin{array}{c}\text { média } \\
(\mathrm{dp})\end{array}$ & \\
\hline Idade (em anos) & 8,04 & 8,05 & 0,984 \\
& $(2,71)$ & $(2,74)$ & \\
\hline Tempo de hospitalização (dias) & 5,26 & $(6,07)$ & $\mathbf{0 , 0 8 1}$ \\
& $(3,32)$ & $(4,61)$ & \\
\hline
\end{tabular}


Tabela 6 - Relação entre a idade e presença de coleções intraperitoneais.

\begin{tabular}{|c|c|c|c|}
\hline \multirow[t]{2}{*}{ Variável } & \multicolumn{2}{|c|}{ Coleção purulenta } & \multirow{2}{*}{$\begin{array}{c}\text { Teste t de } \\
\text { Student (p) }\end{array}$} \\
\hline & $\begin{array}{c}\mathrm{Sim} \\
(\mathrm{n}=113) \\
\text { média } \\
(\mathrm{dp})\end{array}$ & $\begin{array}{c}\text { Não } \\
(\mathrm{n}=187) \\
\text { média } \\
(\mathrm{dp})\end{array}$ & \\
\hline Idade (anos) & $\begin{array}{c}7,52 \\
(2,88)\end{array}$ & $\begin{array}{c}8,36 \\
(2,57)\end{array}$ & 0,010 \\
\hline
\end{tabular}

No hemograma dos pacientes apresentados, a leucocitose estava presente um 83,9\% dos 292 exames realizados com desvio para esquerda em $72,2 \%$, reforçando a importância da associação com a história clínica. $\mathrm{O}$ exame de urina foi um dos fatores de retardo no diagnóstico, quando detectado leucocitúria em $34 \%$ dos pacientes, algumas, tratadas como infecções urinárias. Ressaltamos a associação desse achado laboratorial em casos de apendicites retro-cecais.

O exame radiológico simples de abdome pôde mostrar sinais inespecíficos sobre processos inflamatórios e infecciosos em abdome inferior que, associado à clínica, enfatizou a hipótese diagnóstica. Em alguns casos de crianças em idade pré-escolar esses sinais podem simular imagens sugestivas de processos abdominais obstrutivos ${ }^{15}$.

O estudo ultra-sonográfico tem assumido papel importante nos últimos anos, especialmente nos casos clínicos em que os diagnósticos são duvidosos, referindo-se na literatura índices de acertos de até $93 \%$, podendo em alguns casos prestar informações sobre a severidade do grau de comprometimento apendicular $(67 \%)^{16,17}$. No presente estudo essa modalidade de imagem auxiliou favoravelmente no diagnóstico em cerca de $80,1 \%$ dos casos em que o exame foi indicado.

Inúmeras publicações tem relacionado às várias modalidades de imagens adquiridas com a ultra-sonografia e com a tomografia computadorizada, proporcionando em casos duvidosos de difícil diagnóstico a menor possibilidade de erros e laparotomias desnecessárias ${ }^{18-21}$. Na realidade não se tem um consenso sobre o tema, que na nossa realidade ainda é distante em função do alto custo que proporcionaria ao sistema de saúde.

Optamos pela abordagem com incisão transversa em ponto de McBurney, sendo a maioria dos apêndices cecais encontrados em fase flegmonosa. Nos pacientes em que foram detectadas coleções purulentas localizadas, em pequena quantidade, optou-se por limpeza mecânica da região com gaze seca. Nos casos de coleções de moderadas a grades quantidades, realizaram-se os procedimentos anteriormente descritos associados drenagens peritoneais com drenos de penrose, exteriorizadas por contra-abertura ou pela própria ferida cirúrgica, quando pequena, sem que com isso tenhamos observado maiores complicações infecciosas. A drenagem foi uma maneira de tornar mais segura a vigilância quanto a abscessos intra-cavitários, funcionando também como terapêuticos quando efetivos na sua função, permanecendo por até seis dias em $51,7 \%$ dos pacientes drenados.

As indicações de lavagens de cavidade peritoneal com solução fisiológica aquecida foram restritas aos casos complicados com peritonites supurativas difusas.

Apesar da laparoscopia não ter sido utilizada em nossa casuística, compreendemos tratar-se de uma outra opção terapêutica efetiva quando bem indicada na faixa etária pediátrica, favorecendo a localização fácil e rápida do apêndice cecal, aspirações e lavagens intracavitárias, além da possibilidade do reconhecimento de outras afecções quando do diagnóstico incorreto de apendicite aguda.

Na grande maioria dos casos foi necessária a administração de mais de um tipo de antimicrobiano, tendo-se como a associação mais frequente as que cobriam infecções por agentes Gram-negativos e anaeróbios. Em grande parte dos pacientes esses medicamentos eram mantidos como terapêuticos, tendo-se como critérios para suspensão a ausência de febre, de cateteres e/ou complicações infecciosas, apesar da existência de relatos divergentes quanto ao tipo e tempo de antibioticoterapia e o seu impacto nos parâmetros clínicos $^{22,23}$.

Nos casos de persistência de febre, que em $68,2 \%$ dos casos manteve-se até 48 horas, estando a criança com cobertura antimicrobiana, em acordo as recomendações apresentadas na literatura ${ }^{24}$, recorreu-se à cultura de secreção abdominal colhida no intra-operatório para mudanças dos esquemas inicialmente instituídos.

A média de duração das internações foi de 5,2 dias para os meninos e seis dias para as meninas. Algumas publicações relatam a interferência da idade e grau de comprometimento do apêndice cecal no tempo de hospitalização ${ }^{25}$.

A apendicite aguda na criança, apesar do grande número de casos operados diariamente, continua tendo um grande potencial de complicações resultantes de característi-

Tabela 7 - Relação entre idade e evolução anatomo-patológica do apêndice cecal.

\begin{tabular}{c|ccc|c}
\hline Variável & \multicolumn{3}{|c}{ Fase Evolutiva } & ANOVA(p) \\
\hline & Edematosa & Flegmonosa & Gangrenosa & $(\mathrm{n}=63)$ \\
& $(\mathrm{n}=25)$ & $(\mathrm{n}=212)$ & média & \\
& média & média & $(\mathrm{dp})$ & \\
\hline Idade (anos) & $(\mathrm{dp})$ & $(\mathrm{dp})$ & 7,97 & 0,784 \\
& 8,40 & 8,02 & $(3,03)$ & \\
\hline
\end{tabular}


cas peculiares à idade e às variedades na sua forma de apresentação. Obviamente, com o grande avanço das técnicas de aquisição de imagem e das provas laboratoriais, houve uma diminuição do tempo de elaboração do diagnóstico, reduzindo a mortalidade. Entretanto, a despeito desses fatos, a maioria dos dados estatísticos presentes na literatura, mostram que a morbidade e a mortalidade estão presentes mesmo nos países mais desenvolvidos ${ }^{26,27}$.
Acreditamos que em diagnóstico difíceis, duvidosos, a observação hospitalar é obrigatória, com reavaliações repetidas, se possível pela mesma equipe, até ser descartada ou confirmada a doença, de modo que seja evitado, o que constatamos mesmo nos dias atuais, os retardos diagnósticos, que não só são maléficos ao paciente, como aumentam o custo do atendimento ao sistema de saúde.

\begin{abstract}
Background: In our clinical practice, we have observed that despite the great technological advances in diagnostic methods acute appendicitis still represents a problem in children, resulting in late diagnosis and treatment, and case of greater severity. Ou robjective is to assess the current state of diagnosis and treatment of acute appendicitis in children treated in two important referral hospitals of São Paulo (Brazil), over a 30 month's period. Methods: The variables studied were: age, sex, clinical manifestations, time for the diagnosis to be established, the findings from physical examination and laboratory tests, surgical findings and antibiotic protocols, postoperative complications and hospital lenght of stay. Results: In the present sample, of 300 children, $65 \%$ were boys and $35 \%$ girls, with an initial diagnosis of appendicitis being made in $63 \%$ of the cases. The signs and symptoms most frequently encountered were: abdominal pain in the right iliac fossa (85.3\%) and peritoneal irritation in this region (82\%). We identified leukocytosis in $83 \%$ of the patients and the urine analysis showed leukocyturia in $39.7 \%$ of the patients. Around $92.4 \%$ of the simple radiological studies of the abdomen produced images that were not specific to acute appendicitis. Ultrasonography studies diagnosed the disease in $80.1 \%$ of the cases. Various antibiotic schemes were used, although there was special attention towards Gram-negative and anaerobic bacteria. The main complication was infection of the surgical wound, and there was no mortality. The average hospital length of stay was 5 days. Conclusion: In spite of greater knowledge of acute appendicitis and greater refinement of laboratory and radiological techniques, the diagnosis is still delayed and disease constitutes a cause of great morbidity among pediatric age groups.
\end{abstract}

Key words: Appendicitis; Acute Disease; Appendicitis/diagnosis; Abdomen, acute; Diagnosis techniques, digestive system; Complications; Child.

\section{REFERÊNCIAS}

1. Irish MS, Pearl RH, Caty MG, et al. The approach to common abdominal diagnosis in infants and children. Pediatr Clin North Am. 1998;45(4):729-72.

2. Godquin B. Le centenaire de l'appendicectomie (1887-1987). Chirurgie. 1987;113(4):336-43.

3. O'Toole SJ, Karamanoukian HL, Allen JE, et al. Insurance-related differences in the presentation of pediatric appendicitis. J Pediatr Surg. 1996;31(8):1032-4.

4. Pearl RH, Hale DA, Molloy M, et al. Pediatric appendicectomy. J Pediatr Surg. 1995;30(2):173 -8 discussion 178-81.

5. Newman K, Ponsky T, Kittle K, et al. Appendicitis 2000: variability in practice outcomes, and resource utilization at thirty pediatric hospitals. J Pediatr Surg. 2003;38(2): 378-9.

6. Yardeni D, Hirschl RB, Drongowski RA, et al. Delayed versus immediate surgery in acute appendicitis: do we need to operate during the night? J Pediatr Surg. 2004; 39(3):464-9.

7. Ndungu JM. Appendicitis in African children. East Afr Med J. 1994;71(5):277-8.

8. Eldar S, Nash E, Sabo E, et al. Delay of surgery in acute appendicitis. Am J Surg. 1997;173(3):194-8.

9. Younger. Am J Surg. 1990;159(6):589-92.

10. Massad M, Srouji M, Awdeh A, et al. Neonatal appendicitis: case report and a revised review of the english literature. $\mathrm{Z}$ Kinderchir. 1986;41(4):241-3.

11. Primatesta P, Goldacre MJ. Appendicectomy for acute appendicitis and for other conditions: an epidemiological study. Int J Epidemiol. 1994;23(1):155-60.
12. Fergusson JAF, Hitos K, Simpson E. Utility of white cell count and ultrassound in diagnosis of acute appendicitis. ANZ J Surg. 2002;72(11):781-5.

13. Hallan S, Asberg A, Edna TH. Additional value of biochemical tests in suspected acute appendicitis. Eur J Surg. 1997;163(7):533-8.

14. van den Broek WT, van den Ende ED, Bijner ED, et al. Which children could benefit from additional diagnostic tools in cases of suspected appendicitis? J Pediatr Surg. 2004;39(4):570-4.

15. Hulett R. Imaging of appendicitis. West J Med. 1996;165(1-2):54-5.

16. Galindo Gallego M, Fadrique B, Nieto MA, et al. Evaluation of ultrasonography and clinical diagnostic scoring in suspected appendicitis. Br J Surg.1998;85(1):37-40.

17. Kaneko K, Tsuda M. Ultrasound-based decision making in the treatment of acute appendicitis in children. J Pediatr Surg. 2004;39(9):1316-20.

18. Patrick DA, Janik JE, Janik JS, et al. Increased CT scan utilization does not improve the diagnostic accuracy of appendicitis in children. J Pediatr Surg. 2003; 38(5):659-62.

19. Stephen AE, Segev DL, Ryan DP, et al. The diagnosis of acute appendicitis in a pediatric population: to CT or not CT. J Pediatr Surg. 2003;38(3):367-71.

20. Martin AE, Vollman D, Adler B, et al. CT scans may not reduce the negative appendicectomy rate in children. J Pediatr Surg. 2004;39(6):886-90.

21. Hagendorf BA, Clarke JR, Burd RS. The optimal initial management of children with suspected appendicitis: a decision analysis. J Pediatr Surg. 2004;39(6):880-5.

22. Muehlstedt SG, Pham TQ, Schmeling DJ. The management of pediatric appendicitis: a survey of North America Pediatric Surgeons. J Pediatr Surg. 2004; 39(6):875-9. 
23. Smink DS, Finkelstein JA, Garcia Peña BM, et al. Diagnosis of acute appendicitis in children using a clinical practice guideline. J Pediatr Surg. 2004;39(3):458-63.

24. Bilik R, Burnweit C, Shandling B. Is abdominal cavity culture of any value in appendicitis? Am J Surg. 1998;175(4):267-70.

25. Incesu L. Diagnosis of acute appendicitis in children. AJR Am J Roentgenol. 1995;165(4):1014.

26. Emil S, Laberge JM, Mikhail P, et al. Appendicitis in children: a ten-year update of therapeutic recommendations. J Pediatr Surg. 2003;38(2):236-42.
27. Meier DE, Guzzetta PC, Barber RG, et al. Perforated appendicitis in children: is there a best treatement? J Pediatr Surg. 2003;38(10):1520-4.

Endereço para correspondência:

Prof. Dr. José Luiz Martins

Rua dos Otonis, 131

Vila Clementino - São Paulo - SP.

Cep: 04025 - 000

Fone: 011- 99103351

Fone / Fax: 0XX $11-55628355$

E-mail: jlmartins1@terra.com.br 